\title{
Effects of paliperidone extended release on hostility among Thai patients with schizophrenia
}

\author{
This article was published in the following Dove Press journal: \\ Neuropsychiatric Disease and Treatment \\ 12 January 2017 \\ Number of times this article has been viewed
}

\section{Apichat Jariyavilas ${ }^{\prime}$ \\ Nuntika Thavichachart ${ }^{2}$ \\ Ronnachai Kongsakon ${ }^{3}$ \\ Sunanta Chantakarn ${ }^{4}$ \\ Suwanna Arunpongpaisal ${ }^{5}$ \\ Vasu Chantarasak ${ }^{6}$ \\ Piyadit Jaroensook ${ }^{7}$ \\ Khanogwan Kittiwattanagul ${ }^{8}$ \\ Osot Nerapusee?}

'Srithanya Hospital, Department of Mental Health, Ministry of Public Health, Bangkok, 'Department of Psychiatry, Faculty of Medicine, Chulalongkorn University, Bangkok, ${ }^{3}$ Department of Psychiatry,

Faculty of Medicine, Ramathibodi Hospital, Bangkok, ${ }^{4}$ Department of Psychiatry, Faculty of Medicine, Siriraj Hospital, Bangkok,

${ }^{5}$ Department of Psychiatry, Faculty of Medicine, Khon Kaen University, Khon Kaen, ${ }^{6}$ Somdetchaopraya Institute of Psychiatry, Bangkok, ${ }^{7}$ Prasrimahabhodhi Hospital, Ubon Ratchathani, ${ }^{8}$ Khon Kaen Rajanagarindra Psychiatric Hospital, Khon Kaen, ${ }^{9}$ Medical Affairs, Janssen-Cilag, Bangkok, Thailand
Correspondence: Apichat Jariyavilas Srithanya Hospital, Department of Mental Health, Ministry of Public Health, 47 Tiwanon, Mueang Nonthaburi,

Nonthaburi I0I I0, Thailand

Tel +662525098I

Email a_jariyavilas@yahoo.com
Objective: This open-label prospective study investigated the effects of paliperidone extended release (ER) on hostility in Thai patients with schizophrenia.

Background: Patients diagnosed with schizophrenia may be hostile or exhibit aggressive behavior, which can occasion their admission to psychiatric hospital. Antipsychotic medications are often used to treat hostility and aggression in such patients. Paliperidone ER is effective and well tolerated in the treatment of schizophrenia. However, there are no data available for paliperidone ER with regard to its efficacy on hostility and aggression among Thai patients. This study was a part of the PERFEcT study, a 6-month, open-label, multicenter, multicountry, prospective trial to explore the safety, efficacy, and functionality of paliperidone ER tablets. The current study included only the data obtained from Thai participants.

Materials and methods: Flexible dosing of paliperidone ER in a range of 3-12 $\mathrm{mg} /$ day was used, allowing investigators to adjust the dosage of each subject individually. The 199 Thai patients had a stable Clinical Global Impression - severity score before enrollment. Demographic data were collected at enrollment, and assessments took place at 1, 2, 3, and 6 months postbaseline. The Positive and Negative Syndrome Scale (PANSS) and Personal and Social Performance (PSP) scale were used to evaluate efficacy. In this analysis, we report the findings for the specific PANSS factor P7 (hostility) and the PSP subscale disturbing and aggressive behavior. Data were analyzed using paired $t$-test method to investigate changes in mean PANSS and PSP total and subscale scores. The significance level was set at $P<0.05$.

Results: From a total of 199 Thai patients, 148 patients (74.4\%) participated in all visits. There was a significant reduction in mean scores for all total PANSS measures from 1 month onward compared with baseline, as well as ongoing significant reductions in scores from visit to visit. There was a significant reduction in mean hostility score at 2 months $(P<0.05), 3$ months $(P<0.05)$, and 6 months $(P<0.01)(\mathrm{n}=148)$. For the PSP scale, there was a significant acrossthe-board reduction of mean scores from 3 months onward, including in the disturbing and aggressive behavior subscale $(P<0.001)(n=148)$.

Conclusion: Switching from previously unsuccessful antipsychotic treatments to paliperidone ER may be a useful option to reduce hostility and disturbing behavior in patients with schizophrenia. This study in Thai patients is in line with findings in other countries and cultures concerning the management of hostility in patients with schizophrenia.

Keywords: schizophrenia, hostility, aggression, disturbing behavior, paliperidone ER

\section{Background}

Antipsychotic medication forms the mainstay of treatment for schizophrenia. Patients diagnosed with schizophrenia are occasionally hostile or may exhibit violent behavior, and such behavior is frequently the reason for their admission to psychiatric units, where they sometimes require seclusion or restraint. ${ }^{1,2}$ Various antipsychotic medications, such as clozapine, olanzapine, haloperidol, and risperidone, have been investigated for 
their efficacy in treating hostility and aggression in patients with schizophrenia. ${ }^{1,3}$ Paliperidone (9-OH-risperidone) is the major active metabolite of risperidone, and is approved for use as a long-term antipsychotic treatment for schizophrenia. ${ }^{4}$ A paliperidone extended-release (ER) formulation has been developed that has been shown to be effective and well tolerated in the treatment of schizophrenia. ${ }^{5}$ A number of studies have assessed paliperidone in the context of hostility and aggressive behavior. ${ }^{6-9}$ However, there are no data available for paliperidone ER with regard to its efficacy on hostility and aggression among Thai patients.

The socioeconomic, cultural, political, linguistic, health care, and reimbursement systems in Southeast Asian countries are diverse, as well as being different in comparison with other parts of the world..$^{10}$ To investigate the efficacy of paliperidone ER in Southeast Asian patients with schizophrenia, the PERFEcT study was conducted from June 2007 to April 2009. ${ }^{11,12}$ Derived from the PERFEcT study, these findings describe the efficacy of paliperidone ER on hostility among Thai patients with schizophrenia who had switched from other antipsychotic medications because of lack of efficacy, poor tolerability, or poor compliance.

\section{Materials and methods}

\section{Study design and assessments}

The PERFEcT study was an open-label prospective trial to explore the tolerability, safety, and efficacy of flexibly dosed paliperidone ER in subjects with schizophrenia. ${ }^{11,12}$ Flexible dosing of paliperidone ER in a range of 3-12 $\mathrm{mg}$ /day was used, allowing investigators to adjust the dosage of each subject individually. The recommended dose of paliperidone is $6 \mathrm{mg}$ once daily in the morning, but may start at higher/ lower doses based on clinical judgment. Throughout the study, dose adjustment was done at the investigators' discretion based on clinical response. Demographic data was collected at enrollment together with baseline efficacy data. Efficacy assessments took place at 1, 2, 3, and 6 months postbaseline. Concomitant medications (including benzodiazepines and anticholinergics for treatment of extrapyramidal symptoms, but excluding concomitant antipsychotics) could be administered as long as they were duly documented. Adverse event data were collected throughout the study as the events emerged, and safety assessments were carried out at each scheduled visit. Efficacy was assessed using the Positive and Negative Syndrome Scale (PANSS), ${ }^{13}$ Marder score, ${ }^{14}$ Personal and Social Performance (PSP) scale, ${ }^{15}$ and the Clinical Global Impression - severity (CGI-S) scale. ${ }^{16}$ In this analysis, we focus on the findings for the specific PANSS factor P7 (hostility) and the PSP subscale disturbing and aggressive behavior. PANSS evaluations took place at every scheduled visit, while PSP evaluations were carried out at baseline and after 3 and 6 months.

\section{Subjects}

This study was a part of the PERFEcT study, a 6-month, open-label, multicenter, multicountry, prospective trial to explore the safety, efficacy, and functionality of paliperidone ER tablets. The current study included only the data obtained from the 199 Thai participants. In the overall study, 984 patients meeting the Diagnostic and Statistical Manual of Mental Disorders (DSM)-IV criteria ${ }^{17}$ for schizophrenia who had switched their antipsychotics because of lack of efficacy, tolerability, or compliance were recruited from 61 sites in five countries in Southeast Asia (Philippines, Thailand, Hong Kong, Singapore, and Malaysia). As part of the inclusion criteria, these patients were required to be in a nonacute stage, defined as being established on antipsychotic medication used for the treatment of schizophrenia, and with CGI-S change $\leq 1$ in the 4 weeks prior to enrollment. Patients had been given an adequate dose of an appropriate oral atypical antipsychotic for an adequate period of time prior to enrollment, but the previous treatment was considered unsuccessful, due to one or more of the following reasons: lack of efficacy, lack of tolerability or safety, lack of compliance, and/or "other". Subjects who were taking any oral atypical antipsychotic medication were transitioned to an effective dose of paliperidone ER without the need for titration. Subjects were either cross-tapered or directly switched from their previous atypical antipsychotic medication. The switching strategy depended on investigator judgment. The 199 Thai patients whose results are reported here were recruited from 16 sites in Thailand. All patients who volunteered to take part in the study read and signed the written informed consent form for participation before initiation of any trial-related procedures. The study protocol and related materials were reviewed and approved by the Joint Research Ethical Committee (central ethical committee) and each ethical committee participating site: Maharaj Nakorn Chaingmai; Suansaranrom; Khon Kaen Rajanagarindra; Queen Sawang Wadhana Memorial; Rayong; King Chulalongkorn Memorial; Siriraj; Somdejchaophraya Institute of Psychiatry; Suanprung; Ramathibodi; Buddhachinaraj; Srinakarind Khon Kaen University; Prasrimahabhodi; Prince of Songkhla University; Srithanya; Chachoengsao.

\section{Data analysis}

A paired $t$-test was applied for comparison of the efficacy results at the various time points against the baseline values to investigate statistical significance. For the analyses, the 


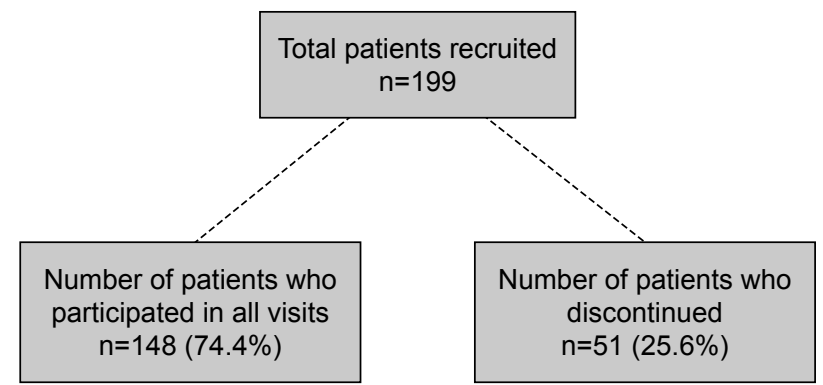

Figure I Disposition of subjects.

level of significance was set at $P<0.05$. SPSS 18.0 (SPSS Inc, Chicago, IL, USA) was used for all statistical procedures.

\section{Results}

Among the 199 Thai patients who entered the study, $106(53.3 \%)$ were male and 93 (46.7\%) female. Of the 199 enrolled patients at the baseline visit, 175 (87.9\%) participated in the second visit (after 1 month), 164 (82.4\%) participated in the third visit (after 2 months), 155 (77.9\%) participated in the fourth visit (after 3 months), and 188 $(94.5 \%)$ took part in the end-of-study evaluation (after 6 months). The data were analyzed from 148 patients (74.4\%) who had recorded P7 in all visits completely, and these patients comprised the group whose results are analyzed here. Stratified by age, 88 patients $(59.5 \%)$ were aged $20-40$ years, 47 patients $(31.8 \%)$ were $41-60$ years old, eight patients $(5.4 \%)$ were aged over 60 years, and five patients (3.4\%) were under 20 years old. Figure 1 shows the disposition of subjects. Table 1 shows patients' demographic and clinical data at baseline for the group who participated in all visits $(\mathrm{n}=148)$.

\section{Efficacy}

Table 2 shows the effects of paliperidone ER treatment on the positive, negative, and general psychopathology scales of the

Table I Demographic and clinical data at baseline

\begin{tabular}{ll}
\hline Characteristic & $\mathbf{n}=\mathbf{I 4 8}$ \\
\hline Age, years & \\
$\quad$ Mean \pm SD & $37.9 \pm 12.2$ \\
$\quad$ Range & $18-79$ \\
Sex, $\mathrm{n}(\%)$ & $77(52)$ \\
$\quad$ Male & $71(48)$ \\
$\quad$ Female & \\
Baseline PANSS, mean \pm SD & $56.6 \pm 19.9$ \\
$\quad$ Overall PANSS score & $1.43 \pm 0.88$ \\
$\quad$ Hostility (P7) & \\
Baseline PSP scale, mean \pm SD & $67.5 \pm 17.7$ \\
$\quad$ Overall PSP score & $1.68 \pm 0.95$ \\
$\quad$ Disturbing and aggressive behavior & $3.37 \pm 1.19$ \\
Baseline CGI-S, mean \pm SD
\end{tabular}

Abbreviations: SD, standard deviation; PANSS, Positive and Negative Syndrome Scale; PSP, Personal and Social Performance; CGI-S, Clinical Global Impression - severity.

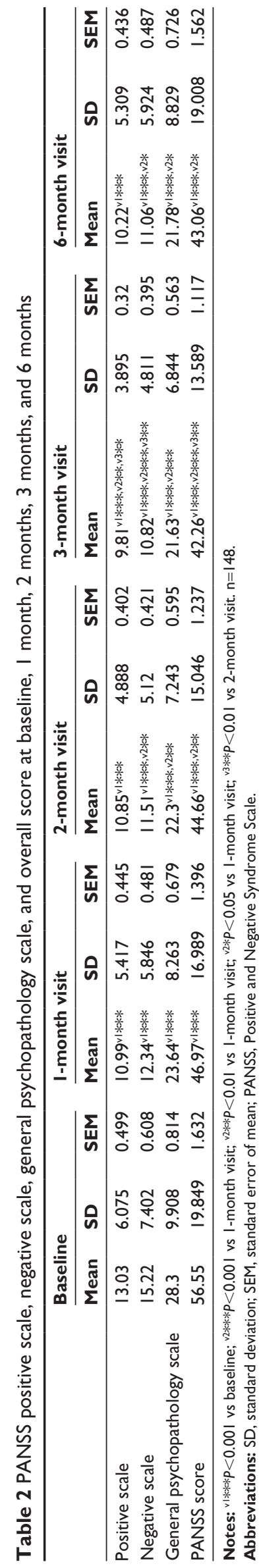


Table 3 Positive and Negative Syndrome Scale P7 (hostility) results

\begin{tabular}{|c|c|c|c|c|c|c|c|c|c|c|c|c|c|c|}
\hline \multicolumn{3}{|c|}{ Baseline } & \multicolumn{3}{|c|}{ I-month visit } & \multicolumn{3}{|c|}{ 2-month visit } & \multicolumn{3}{|c|}{ 3-month visit } & \multicolumn{3}{|c|}{ 6-month visit } \\
\hline Mean & SD & SEM & Mean & SD & SEM & Mean & SD & SEM & Mean & SD & SEM & Mean & SD & SEM \\
\hline $\mathrm{I} .43$ & 0.881 & 0.072 & 1.34 & 0.923 & 0.076 & $1.23^{v 1 *}$ & 0.64 & 0.053 & $1.24^{v 1 *}$ & 0.587 & 0.048 & $1.24^{\mathrm{v} 1 * *}$ & 0.706 & 0.058 \\
\hline
\end{tabular}

Notes: $v 1 * * P<0.01$ vs baseline; ${ }^{v 1 * P}<0.05$ vs baseline. $n=148$.

Abbreviations: SD, standard deviation; SEM, standard error of mean.

PANSS, as well as the overall PANSS scores at the various time points of the study. There was a significant reduction in mean scores for all scales from 1 month onward compared with baseline, as well as ongoing significant reductions in scores from visit to visit. The data were also broken down by individual PANSS subscales, and the P7 (hostility) subscale is reported in Table 3. Compared with baseline, the results showed a significant reduction in mean \pm standard deviation (SD) for the P7 (hostility) score from $1.43 \pm 0.881$ to $1.23 \pm 0.64$ after 2 months $(P<0.05), 1.24 \pm 0.587$ after 3 months $(P<0.05)$, and $1.24 \pm 0.706$ after 6 months $(P<0.01)(\mathrm{n}=148)$. Table 4 shows the effects on the various PSP subscales at the different study time points. Again, there was an acrossthe-board reduction in mean scores from 3 months onward. For the "Disturbing and aggressive behavior" subscale, compared with baseline, mean \pm SD scores were reduced significantly after 3 months from $1.68 \pm 0.948$ to $1.29 \pm 0.538$ $(P<0.001)$, and after 6 months the score had reduced to $1.22 \pm 0.593(P<0.001)(\mathrm{n}=148)$. Table 5 shows the CGI-S scale at different time points. Compared with a mean \pm SD baseline score of $3.37 \pm 1.185$, the scores reduced significantly to $2.69 \pm 1.234$ after 1 month $(P<0.001), 2.6 \pm 1.217$ after 2 months $(P<0.001), 2.44 \pm 1.089$ after 3 months $(P<0.001)$, and $2.31 \pm 1.234$ after 6 months $(P<0.001)(\mathrm{n}=148)$. There were also significant ongoing reductions in the CGI-S score from visit to visit.

\section{Discussion}

This study examined the efficacy of paliperidone ER on hostility and disturbing/aggressive behavior in patients with schizophrenia using the PANSS and PSP scale. In 148 Thai patients with schizophrenia who had switched their antipsychotic medication, there was a significant reduction in mean PANSS P7 (hostility) score after 2 months $(P<0.05)$, 3 months $(P<0.05)$, and 6 months $(P<0.01)$ compared with baseline. This goes alongside a significant reduction in mean scores for all PANSS subscales and the CGI-S scale from 1 month onward and PSP subscales (including disturbing and aggressive behavior) from 3 months onward compared with baseline, as well as ongoing significant reductions in scores from visit to visit.

While the efficacy and safety of paliperidone ER have been demonstrated in various international patient groups, ${ }^{5,6,8,9}$ there are no data available for Thai patients specifically. Thailand, in common with other Southeast Asian countries, has different socioeconomic, cultural, political, linguistic, health care, and reimbursement systems compared with other parts of the world. ${ }^{10}$ These results thus provide a valuable understanding of the role of paliperidone ER in the Thai context.

The results reported here may be compared with those of a 2007 European study, ${ }^{18}$ in which 294 patients with schizophrenia were flexibly dosed with oral paliperidone ER 3-12 mg/day in a naturalistic setting. In that study, significant improvements in PANSS factors (including hostility), PSP, and CGI-S were observed. Baseline PANSS for uncontrolled hostility/ excitement was 10.6 \pm 3.7 . After treatment with paliperidone ER, this reduced to $7.5 \pm 3.4(P<0.0001)$. In common with many pivotal studies, subjects in the European study were treated for 6 weeks, whereas in the results reported here a 6-month treatment period was applied, which might better provide longer-term data on efficacy and tolerability in Thai

Table 4 Personal and Social Performance scale results at baseline, 3-month visit, and 6-month visit

\begin{tabular}{|c|c|c|c|c|c|c|c|c|c|c|}
\hline \multirow{2}{*}{$\begin{array}{l}\text { Personal and social } \\
\text { performance domain }\end{array}$} & \multirow[t]{2}{*}{$\mathbf{n}$} & \multicolumn{3}{|c|}{ Baseline } & \multicolumn{3}{|c|}{ 3-month visit } & \multicolumn{3}{|l|}{ 6-month visit } \\
\hline & & Mean & SD & SEM & Mean & SD & SEM & Mean & SD & SEM \\
\hline Socially useful activities & 148 & 2.22 & 1.2 & 0.099 & $1.64^{v 1 * * *}$ & 0.85 & 0.07 & $1.56^{v 1 * * *}$ & 0.898 & 0.074 \\
\hline Personal and social relationships & 148 & 2.28 & 1.178 & 0.097 & $1.67^{v 1 * * *}$ & 0.876 & 0.072 & $1.534^{\mathrm{v} 1 * * *, v 4 *}$ & 0.884 & 0.073 \\
\hline Self-care & 148 & 1.60 & 0.975 & 0.08 & $1.3^{v 1 * * *}$ & 0.687 & 0.056 & $1.27 \mathrm{vl} * * *$ & 0.706 & 0.058 \\
\hline Disturbing and aggressive behavior & 148 & 1.68 & 0.948 & 0.078 & $1.29 v 1 * * *$ & 0.538 & 0.044 & $1.22^{\mathrm{v} 1 * * *}$ & 0.593 & 0.049 \\
\hline Overall score & 148 & 67.52 & 17.665 & $\mathrm{I} .452$ & $77.82^{v 1 * * *}$ & 14.218 & 1.169 & $81.6^{v 1 * * *, v 4 * * *}$ & 15.499 & 1.274 \\
\hline
\end{tabular}

Notes: ${ }^{v 1 * * *} P<0.001$ vs baseline; ${ }^{v 4 * * * P}<0.001$ vs 3 -month visit; ${ }^{v 4 * P}<0.05$ vs 3 -month visit.

Abbreviations: SD, standard deviation; SEM, standard error of mean. 
Table 5 Clinical Global Impression - severity score at baseline, I month, 2 months, 3 months, and 6 months

\begin{tabular}{|c|c|c|c|c|}
\hline CGI-S & $\mathbf{n}$ & Mean & SD & SEM \\
\hline Baseline & 148 & 3.37 & 1.185 & 0.097 \\
\hline I-month visit & 148 & $2.69^{\vee} 1 * * *$ & $\mathrm{I} .234$ & 0.101 \\
\hline 2-month visit & 148 & $2.60^{\vee 1 * * *}$ & 1.217 & 0.1 \\
\hline 3-month visit & 148 & $2.44^{\mathrm{v} 1 * * *, \mathrm{v} 2 * *}$ & 1.089 & 0.09 \\
\hline 6-month visit & 148 & $\left.2.3\right|^{\mathrm{v} 1 * * *, \mathrm{v} 2 * * *, v 3 * * *}$ & 1.234 & 0.101 \\
\hline
\end{tabular}

patients. The patients in the European study were experiencing an acute exacerbation of schizophrenia, which may explain their relatively higher baseline PANSS score.

Limitations of this study include firstly its open-label design and lack of a comparator group. However, this reflects more accurately the clinical situation in a real-world setting, and the assessment criteria are objective, detailed, and standard in clinical trials of antipsychotic medication. Furthermore, this study permitted patients to use concomitant medications, which are often disallowed in randomized controlled trails. A second limitation is that validated psychopathological rating scales are often difficult to apply in a naturalistic setting, even though rater training was given.

\section{Conclusion}

In summary, this study in Thai patients with schizophrenia confirms the finding that paliperidone ER is effective in treating hostility and disturbing/aggressive behavior, and as this was an open-label study with flexible dosing, it could reflect the real-life situation in typical clinical practice in Thailand.

Switching from previously unsuccessful antipsychotic treatments to paliperidone ER may be a useful option to reduce hostility and disturbing behavior in patients with schizophrenia. This study in Thai patients is in line with findings in other countries and cultures concerning the management of hostility in patients with schizophrenia.

\section{Acknowledgment}

This work was part of the PERFEcT study, which was funded by Janssen-Cilag.

\section{Disclosure}

$\mathrm{ON}$ is an employee of Janssen-Cilag Thailand. The other authors report no conflicts of interest in this work.

\section{References}

1. Citrome L, Volavka J, Czobor P, et al. Effects of clozapine, olanzapine, risperidone, and haloperidol on hostility among patients with schizophrenia. Psychiatr Serv. 2001;52(11):1510-1514.

2. Steinert T, Bergbauer G, Schmid P, Gebhardt RP. Seclusion and restraint in patients with schizophrenia: clinical and biographical correlates. J Nerv Ment Dis. 2007;195(6):492-496.

3. Aleman A, Kahn RS. Effects of the atypical antipsychotic risperidone on hostility and aggression in schizophrenia: a meta-analysis of controlled trials. Eur Neuropsychopharmacol. 2001;11(4):289-293.

4. MIMS [website on the Internet]. Available from: http://www.mims.com/ Thailand/drug/info/Invega/Invega?type=brief. Accessed October 17, 2016.

5. Janicak PG, Winans EA. Paliperidone ER: a review of the clinical trial data. Neuropsychiatr Dis Treat. 2007;3(6):869-897.

6. Meltzer HY, Bobo WV, Nuamah IF, et al. Efficacy and tolerability of oral paliperidone extended-release tablets in the treatment of acute schizophrenia: pooled data from three 6-week, placebo-controlled studies. J Clin Psychiatry. 2008;69(5):817-829.

7. Canuso CM, YoussefEA, Bossie CA, Turkoz I, Schreiner A, Simpson GM Paliperidone extended-release tablets in schizophrenia patients previously treated with risperidone. Int Clin Psychopharmacol. 2008; 23(4):209-215.

8. Davidson M, Emsley R, Kramer M, et al. Efficacy, safety and early response of paliperidone extended-release tablets (paliperidone ER): results of a 6-week, randomized, placebo-controlled study. Schizophr Res. 2007;93(1-3):117-130.

9. Dirks B, Youssef E, Bossie C, Turkoz I, Canuso C. Effects of paliperidone ER in patients with schizophrenia previously treated with olanzapine Poster presented at: 2007 International Congress of Schizophrenia Research; March 28-April 1, 2007; Colorado Springs, CO.

10. Tan CH, Shinfuku N, Sim K. Psychotropic prescription practices in East Asia: looking back and peering ahead. Curr Opin Psychiatry. 2008; 21(6):645-650.

11. Thavichachart N, Kongsakon R, Lo WT, et al. The psychopathological characteristics of treatment discontinuation group in 6-month treatment with paliperidone ER. Int J Clin Pract. 2012;66(10):969-975.

12. Suttajit S, Arunpongpaisal S, Srisurapanont M, et al. Psychosocial functioning in schizophrenia: are some symptoms or demographic characteristics predictors across the functioning domains? Neuropsychiatr Dis Treat. 2015;11:2471-2477.

13. Kay SR, Flszbein A, Opfer LA. The Positive and Negative Syndrome Scale (PANSS) for schizophrenia. Schizophr Bull. 1987;13(2): 261-276.

14. Marder SR, Davis JM, Chouinard G. The effects of risperidone on the five dimensions of schizophrenia derived by factor analysis: combined results of the North American trials. J Clin Psychiatry. 1997;58(12): 538-546.

15. Nasrallah H, Morosini P, Gagnon DD. Reliability, validity and ability to detect change of the Personal and Social Performance scale in patients with stable schizophrenia. Psychiatry Res. 2008;161(2):213-224.

16. Guy W, Bonato R. Manual for the ECDEU Assessment Battery. 2nd ed. Bethesda (MD): National Institute of Mental Health; 1970.

17. American Psychiatric Association. Diagnostic and Statistical Manual of Mental Disorders. 4th ed. Arlington (VA): APA; 1994.

18. Schmauss M, Jukić V, Siracusano A, et al. Flexible dosing with paliperidone ER in the treatment of patients with acutely exacerbated schizophrenia: results from a single-arm, open-label study. Curr Med Res Opin. 2012;28(8):1395-1404. 


\section{Publish your work in this journal}

Neuropsychiatric Disease and Treatment is an international, peerreviewed journal of clinical therapeutics and pharmacology focusing on concise rapid reporting of clinical or pre-clinical studies on a range of neuropsychiatric and neurological disorders. This journal is indexed on PubMed Central, the 'PsycINFO' database and CAS,

and is the official journal of The International Neuropsychiatric Association (INA). The manuscript management system is completely online and includes a very quick and fair peer-review system, which is all easy to use. Visit http://www.dovepress.com/testimonials.php to read real quotes from published authors.

Submit your manuscript here: http://www.dovepress.com/neuropsychiatric-disease-and-treatment-journal 